\title{
Multi-rate Tracking Control for a Space Robot on a Controlled Satellite: A Passivity-based Strategy
}

\author{
Marco De Stefano ${ }^{1,2}$, Hrishik Mishra ${ }^{1}$, Ribin Balachandran ${ }^{1}$, Roberto Lampariello ${ }^{1}$, \\ Christian $\mathrm{Ott}^{1}$, Senior Member, IEEE and Cristian $\mathrm{Secchi}^{2}$, Senior Member, IEEE
}

\begin{abstract}
In this work we design a novel control strategy for a space manipulator operating on a controlled base. The proposed controllers resolve the tracking of the end-effector and the regulation of the base. In particular, we focus on the effects due to the different sampling rates of the manipulator and the base controllers which can generate stability issues. These effects are analysed from an energetic perspective and passivity-based controllers are designed for the base and the manipulator to avoid instability. The method is validated with simulations and experiments on a robotic facility, the OOS-Sim.
\end{abstract}

Index Terms-Space Robotics and Automation, Compliance and Impedance Control, Multi-Robot Systems, Tracking Control.

\section{INTRODUCTION}

$\mathbf{I}$ $\mathrm{N}$ On-Orbit Servicing (OOS) and active space-debris removal missions, the approach phase concerns the tracking of a free-floating target (defunct satellite) with a free-flying space robot (i.e. a robotic manipulator on a fully actuated base in a gravity-free environment) while maintaining the base positioning within some limits [1].

Space robot control algorithms have been developed over the years for the approach phase. Usually, an impedance controller for the end-effector is implemented because the manipulator might interact with the floating target satellite pushing it away and compromising the mission [2]. The impedance control problem of a free-floating robot was treated in [3] and [4] using feedback linearization. In [5], a control based on a generalized Jacobian matrix is proposed and in [6] a sensory feedback control scheme is discussed to deal with the floating nature of the base. However in these works the base was not controlled. In the direction of coordinated control, i.e. the control of the base and manipulator simultaneously, [7] proposes a momentum-based strategy with reaction wheels and [8] introduces a coordinated controller based on feedback linearization. For the tracking case, a passivity-based tracking controller was proposed in [9], however the tracking was

Manuscript received: September, 10, 2018; Revised December, 05, 2018; Accepted January, 9, 2019.

This paper was recommended for publication by Editor Jonathan Roberts upon evaluation of the Associate Editor and Reviewers' comments.

${ }^{1}$ Marco De Stefano, Hrishik Mishra, Ribin Balachandran, Roberto Lampariello and Christian Ott are with the Institute of Robotics and Mechatronics, German Aerospace Center (DLR), 82234 Wessling, Germany (email: marco.destefano@dlr.de; hrishik.mishra@dlr.de; ribin.balachandran@dlr.de; roberto.lampariello@dlr.de; christian.ott@dlr.de).

${ }^{2}$ Marco De Stefano and Cristian Secchi are with the University of Modena and Reggio Emilia, 41100 Modena, Italy (email: marco.destefano@dlr.de; cristian.secchi@unimore.it).

Digital Object Identifier (DOI): 10.1109/LRA.2019.2895420

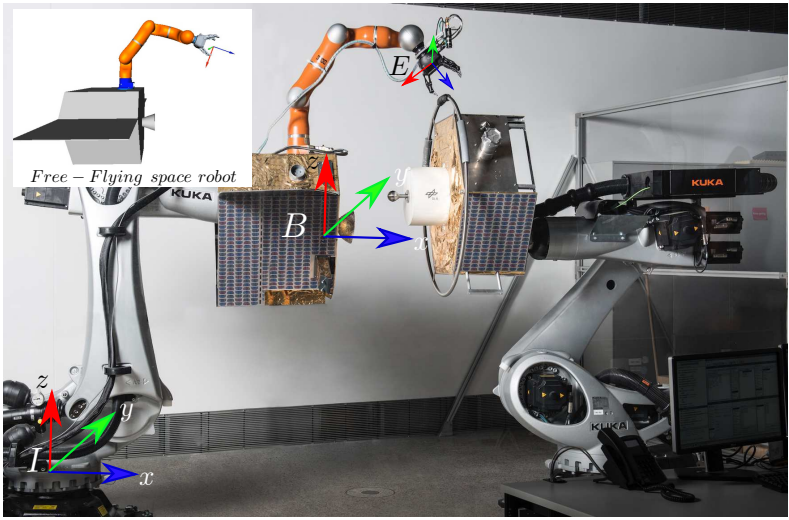

Fig. 1: The OOS-Sim facility: free-flying space robot (left) and target satellite-robot (right). The industrial robots simulate the dynamics of satellite and the Light-Weight-Robot is the manipulation arm of the free-flying space robot. $I$ is the inertial frame, $B$ the base frame and $E$ is the end-effector frame.

performed in joint space and for a fixed-base robot. In [10], a trajectory tracking problem is addressed for a space robot, but, with an unactuated base.

Although the regulation and tracking problem of a freeflying space robot has been addressed previously in literature [4], [7], [8], [11], the problems specific to the multi-rate control were not discussed. In [12, §3.2.2], the problem associated with relative rates between the control of the base and robotic arm (for the approach and capture phase) has been underlined as an important factor to be considered for a space mission. Indeed, due to base actuation and GNC (Guidance Navigation and Control) system constraints, the control of the base needs to run at low rate (between $1 \mathrm{~Hz}$ and $10 \mathrm{~Hz}$ ) and the controller of the manipulator at higher rate (usually $1000 \mathrm{~Hz}$ ) [13]. Therefore, discrete controllers and multi-rate effects must be taken into account during the design of the controllers (see e.g. [14]) because they can jeopardize the stability of the system [15]. Within this context, a first approach was developed in our previous work [16] in which, however, only the regulation problem for the manipulator and its base was treated and this might not be suitable for a trajectory-tracking control needed in the approach phase.

In this paper, we develop a passivity-based coordinated controller for a free-flying space robot. Besides guaranteeing trajectory tracking for the manipulator and regulation for the base, we address the multi-rate nature of the controllers from an energetic perspective. To this end, we will exploit the time-domain passivity approach [17] that has already been 
successfully exploited for coping with non-idealities in space robotics applications (see e.g. [18] and [19]).

The contribution of the paper is threefold. First, we design a tracking control for the end-effector and a regulation control for its base and we prove stability in continuous-time. Second, we show how different sampling rates of the two controllers can interfere and how this can lead to instability. Therefore, two passivity controllers, one for the manipulator and one for the controlled base, are designed for ensuring passivity. Third, we validate the controllers in a simulation study and we verify it on a robotic facility, the OOS-Sim [2] shown in Fig. 1, which is able to simulate the dynamics of a freeflying robot on-ground ${ }^{1}$. The paper is structured as follows: Sec. II introduces the model of the free-flying robot and the combined control laws are presented in Sec. III. The problem statement in using controllers with different sampling rate is given in Sec. III-B. Sec. IV shows how to render the discretetime controllers passive. Validation results are shown in Sec. V with experiments in Sec. VI. Sec. VII concludes the paper.

\section{FREE-FLyING RoBOT MODEL}

The general equations of motion of a gravity-free robot with $n$ joints mounted on a moving base are defined as [20]:

$$
\begin{aligned}
{\left[\begin{array}{cc}
H_{b} & H_{b m} \\
H_{b m}^{T} & H_{m}
\end{array}\right]\left[\begin{array}{c}
\ddot{x}_{b} \\
\ddot{q}
\end{array}\right]+} \\
{\left[\begin{array}{cc}
C_{b} & C_{b m} \\
C_{m b} & C_{m}
\end{array}\right]\left[\begin{array}{c}
\dot{x}_{b} \\
\dot{q}
\end{array}\right]=\left[\begin{array}{c}
F_{b} \\
\tau
\end{array}\right], }
\end{aligned}
$$

where $\boldsymbol{H}_{\boldsymbol{b}} \in \mathbb{R}^{6 \times 6}, \boldsymbol{H}_{\boldsymbol{m}} \in \mathbb{R}^{n \times n}, \boldsymbol{H}_{\boldsymbol{b} \boldsymbol{m}} \in \mathbb{R}^{6 \times n}$ are the inertia matrices of the whole system, manipulator and the coupling between the base and the manipulator, respectively ${ }^{2}$. $\boldsymbol{C}_{\boldsymbol{b}} \in \mathbb{R}^{6 \times 6}, \boldsymbol{C}_{\boldsymbol{m}} \in \mathbb{R}^{n \times n}$ and $\boldsymbol{C}_{\boldsymbol{b} \boldsymbol{m}} \in \mathbb{R}^{6 \times n}$ are the nonlinear Coriolis/centrifugal matrix of the base, manipulator and coupling between base-manipulator, respectively. The vectors $\ddot{\boldsymbol{x}}_{\boldsymbol{b}} \in \mathbb{R}^{6}$ and $\ddot{\boldsymbol{q}} \in \mathbb{R}^{n}$ are the acceleration of the base (linear and angular) and the acceleration of the robot joints respectively and, $\dot{\boldsymbol{x}}_{\boldsymbol{b}} \in \mathbb{R}^{6}$ and $\dot{\boldsymbol{q}} \in \mathbb{R}^{n}$ are the respective velocity vectors. $\boldsymbol{F}_{\boldsymbol{b}} \in \mathbb{R}^{6}$ is the force-torque wrench acting on the center of mass of the base-body and $\tau \in \mathbb{R}^{n}$ is the input torque vector to the manipulator.

The end-effector Cartesian velocity $\dot{\boldsymbol{x}}_{\boldsymbol{m}} \in \mathbb{R}^{6}$ is given by:

$$
\dot{x}_{m}=J_{b} \dot{x}_{b}+J_{m} \dot{q}
$$

where $\boldsymbol{J}_{\boldsymbol{b}} \in \mathbb{R}^{6 \times 6}$ and $\boldsymbol{J}_{\boldsymbol{m}} \in \mathbb{R}^{6 \times n}$ are the Jacobian matrices of the base and manipulator, respectively. Equation (1) can be expressed with a proper change of coordinates from $\xi_{q}$ to $\xi_{m}$, where:

$$
\xi_{q}=\left[\begin{array}{c}
\dot{x}_{b} \\
\dot{q}
\end{array}\right], \xi_{m}=\left[\begin{array}{c}
\dot{x}_{b} \\
\dot{x}_{m}
\end{array}\right] .
$$

The relationship between the new coordinate $\xi_{m}$ with $\xi_{q}$ are given by the Jacobian $\tilde{\boldsymbol{J}}$ defined as follows:

$$
\xi_{m}=\underbrace{\left[\begin{array}{cc}
I & 0 \\
J_{b} & J_{m}
\end{array}\right]}_{\tilde{J}} \xi_{q} .
$$

\footnotetext{
${ }^{1}$ Experiments can be found also in the video attached to this paper.

${ }^{2}$ Throughout the paper the quantities with subscript $m$ are pertinent to manipulator and quantities with subscript $b$ to the base
}

We assume that the manipulator is not redundant and that singular configurations are avoided using, e.g., a motion planner [10], therefore,

$$
\tilde{J}^{-T}=\left[\begin{array}{cc}
I & -J_{b}^{T} J_{m}^{-T} \\
0 & J_{m}^{-T}
\end{array}\right]
$$

Now, using the relationship in (4), the general equation (1) can be rewritten as:

$$
\begin{gathered}
\underbrace{\left[\begin{array}{cc}
M_{b} & M_{b m} \\
M_{b m}^{T} & M_{m}
\end{array}\right]}_{M}\left[\begin{array}{c}
\ddot{x}_{b} \\
\ddot{x}_{m}
\end{array}\right]+\underbrace{\left[\begin{array}{cc}
\Gamma_{b} & \Gamma_{b m} \\
\Gamma_{m b} & \Gamma_{m}
\end{array}\right]}_{\Gamma}\left[\begin{array}{c}
\dot{x}_{b} \\
\dot{x}_{m}
\end{array}\right] \\
=\tilde{J}^{-T}\left[\begin{array}{c}
F_{b} \\
\tau
\end{array}\right]=\left[\begin{array}{c}
F_{b}-J_{b}^{T} F_{m} \\
F_{m}
\end{array}\right], \quad(6)
\end{gathered}
$$

where $\boldsymbol{M} \in \mathbb{R}^{12 \times 12}$ and $\boldsymbol{\Gamma} \in \mathbb{R}^{12 \times 12}$ are the inertia and Coriolis/centrifugal matrices in the new coordinate system. $\boldsymbol{F}_{\boldsymbol{m}} \in \mathbb{R}^{6}$ is the Cartesian wrench at the end-effector defined as $\boldsymbol{F}_{\boldsymbol{m}}=\boldsymbol{J}_{\boldsymbol{m}}^{-\boldsymbol{T}} \boldsymbol{\tau}$.

The system (6) is a fully actuated Euler-Lagrange system and therefore, see e.g. [21], it is passive with respect to the generalized force-velocity pair $\left(\left(\dot{x}_{b}^{T}, \dot{x}_{m}^{T}\right)^{T},\left(\left(F_{b}-\right.\right.\right.$ $\left.\left.\left.J_{b}^{T} F_{m}\right)^{T}, F_{m}^{T}\right)^{T}\right)$.

\section{Controllers in Continuous And Discrete Time}

The goal of the controller is to resolve a tracking problem for the end-effector and regulation of its base. To achieve this goal, the controller at the base will be function of the end-effector desired trajectory. This is in contrast with [16] where only regulation control was implemented for both the base and end-effector and therefore, the tracking terms did not appear. However, this is a natural consequence of the coupled dynamics between base and end-effector motions and must be taken into account in the design of the control laws. In this section, first the controllers are presented in continuoustime with stability proofs. Later, the effects of the controllers running at different sampling rates are discussed.

\section{A. Coordinated Control in Continuous Time}

The continuous-time tracking control for the end-effector is designed as follows:

$$
F_{m}=M_{m} \ddot{x}_{m d}+\Gamma_{m} \dot{x}_{m d}-K_{P m} \Delta x_{m}-K_{D m} \Delta \dot{x}_{m}
$$

where $\ddot{\boldsymbol{x}}_{\boldsymbol{m} \boldsymbol{d}}(\boldsymbol{t}) \in \mathbb{R}^{6}$ and $\dot{\boldsymbol{x}}_{\boldsymbol{m} \boldsymbol{d}}(\boldsymbol{t}) \in \mathbb{R}^{6}$ are respectively the desired acceleration and velocity of the manipulator endeffector expressed in the inertial frame. Usually, such information can be generated by a motion planner which provides a feasible trajectory for the end-effector [10]. $\boldsymbol{K}_{\boldsymbol{P} \boldsymbol{m}}, \boldsymbol{K}_{\boldsymbol{D m}} \in$ $\mathbb{R}^{6 \times 6}$ are stiffness and damping matrices respectively. The vector $\Delta \boldsymbol{x}_{\boldsymbol{m}}=\left(\boldsymbol{x}_{\boldsymbol{m}}-\boldsymbol{x}_{\boldsymbol{m} \boldsymbol{d}}\right) \in \mathbb{R}^{6}$ is the error between the measured and desired poses (position and orientation) of the manipulator end-effector. Similarly, the vector $\Delta \dot{x}_{m}=$ $\left(\dot{\boldsymbol{x}}_{\boldsymbol{m}}-\dot{\boldsymbol{x}}_{\boldsymbol{m} \boldsymbol{d}}\right)$ is the error between the measured and desired end-effector velocities. The controller in (7) can be projected into the joint space to have torque command to the manipulator as: $\boldsymbol{\tau}=\boldsymbol{J}_{\boldsymbol{m}}^{T} \boldsymbol{F}_{\boldsymbol{m}}$. 
For the base, the regulation control is designed as follows: $F_{b}=M_{b m} \ddot{x}_{m d}+\Gamma_{b m} \dot{x}_{m d}-K_{P b} \Delta x_{b}-K_{D b} \dot{x}_{b}+J_{b}^{T} F_{m}$

where $\boldsymbol{K}_{\boldsymbol{P b}}, \boldsymbol{K}_{\boldsymbol{D b}} \in \mathbb{R}^{6 \times 6}$ are stiffness and damping matrices of the regulation control of the base, respectively. The vector $\Delta \boldsymbol{x}_{\boldsymbol{b}}=\left(\boldsymbol{x}_{\boldsymbol{b}}-\boldsymbol{x}_{\boldsymbol{b d}}\right) \in \mathbb{R}^{6}$ is the error (in position and orientation) between the measured and desired base pose. Notice that the orientation error for the base and manipulator exploits the quaternion representation calculated from the relative orientation matrix error (see [16] for more details).

It is worth pointing out that in (8), although the base is regulating about a set-point, the control law is a function of the desired end-effector velocity and acceleration. This is due to the inertial coupling between the base and endeffector tracking motions. Therefore, the regulation control law proposed in [16] for the base controller will not be suitable. Otherwise it might result in instability during the tracking. It can be verified that

$$
\left[\Delta \dot{x}_{b}, \Delta \dot{x}_{m}, \Delta x_{b}, \Delta x_{m}\right]=0
$$

is an equilibrium point of (6) using (7) and (8), and that the following proposition holds true. Note that the controller at the base resolves only the regulation problem, therefore the velocity error in (9) results to be $\Delta \dot{\boldsymbol{x}}_{\boldsymbol{b}}=\dot{\boldsymbol{x}}_{\boldsymbol{b}}$.

Proposition 1: Consider the system (6), with the tracking control law (7) for the end-effector and the regulation control law (8) for the base. Then, the equilibrium point in (9) is asymptotically stable.

Proof 1: Consider the total positive definite energy of the system, which is upper and lower bounded, as the following candidate Lyapunov function:

$$
\begin{aligned}
& V=\frac{1}{2}\left[\begin{array}{ll}
\dot{x}_{b}^{T} & \Delta \dot{x}_{m}^{T}
\end{array}\right] M\left[\begin{array}{c}
\dot{x}_{b} \\
\Delta \dot{x}_{m}
\end{array}\right]+ \\
& \frac{1}{2}\left[\begin{array}{ll}
\Delta x_{b}^{T} & \Delta x_{m}^{T}
\end{array}\right] \underbrace{\left[\begin{array}{cc}
K_{P b} & 0 \\
0 & K_{P m}
\end{array}\right]}_{K} \underbrace{\left[\begin{array}{c}
\Delta x_{b} \\
\Delta x_{m}
\end{array}\right]}_{\Delta x},
\end{aligned}
$$

where $\boldsymbol{M}$ and $\boldsymbol{K}$ are positive definite matrices. Computing the time-derivative, we obtain:

$$
\begin{aligned}
& \dot{V}=\left[\begin{array}{ll}
\dot{x}_{b}^{T} & \Delta \dot{x}_{m}^{T}
\end{array}\right][M\left[\begin{array}{c}
\ddot{x}_{b} \\
\ddot{x}_{m}-\ddot{x}_{m d}
\end{array}\right]+\frac{1}{2} \dot{M} \underbrace{\left[\begin{array}{c}
\dot{x}_{b} \\
\Delta \dot{x}_{m}
\end{array}\right]}_{\Delta \dot{x}} \\
& \left.+\boldsymbol{K}\left[\begin{array}{c}
\Delta x_{b} \\
\Delta x_{m}
\end{array}\right]\right] .
\end{aligned}
$$

Substituting the values $M\left[\begin{array}{ll}\ddot{x}_{b}^{T} & \ddot{x}_{m}^{T}\end{array}\right]^{T}$ from (6), we get:

$$
\begin{aligned}
\dot{V}=\left[\begin{array}{ll}
\dot{x}_{b}^{T} & \Delta \dot{x}_{m}^{T}
\end{array}\right]-\Gamma\left[\begin{array}{c}
\dot{x}_{b} \\
\dot{x}_{m}
\end{array}\right]+\left[\begin{array}{c}
F_{b}-J_{m}^{T} F_{m} \\
F_{m}
\end{array}\right]- \\
M\left[\begin{array}{c}
0 \\
\ddot{x}_{m d}
\end{array}\right]+\frac{1}{2} \dot{M}\left[\begin{array}{c}
\dot{x}_{b} \\
\Delta \dot{x}_{m}
\end{array}\right]+ \\
\left.\Gamma\left[\begin{array}{c}
0 \\
\dot{x}_{m d}
\end{array}\right]-\Gamma\left[\begin{array}{c}
0 \\
\dot{x}_{m d}
\end{array}\right]+K\left[\begin{array}{c}
\Delta x_{b} \\
\Delta x_{m}
\end{array}\right]\right]
\end{aligned}
$$

and considering the skew-symmetric property of the EulerLagrange system, where:

$$
\left[\begin{array}{ll}
\dot{\boldsymbol{x}}_{\boldsymbol{b}}^{\boldsymbol{T}} & \Delta \dot{\boldsymbol{x}}_{\boldsymbol{m}}^{\boldsymbol{T}}
\end{array}\right] \frac{1}{2}(\dot{\boldsymbol{M}}-2 \boldsymbol{\Gamma})\left[\begin{array}{c}
\dot{\boldsymbol{x}}_{\boldsymbol{b}} \\
\Delta \dot{\boldsymbol{x}}_{\boldsymbol{m}}
\end{array}\right]=0,
$$

equation (12) can be simplified and rewritten as:

$$
\begin{array}{r}
\dot{V}=\dot{x}_{b}^{T} F_{b}-\dot{x}_{b}^{T} J_{b}^{T} F_{m}+\Delta \dot{x}_{m}^{T} F_{m}-\dot{x}_{b}^{T} M_{b m} \ddot{x}_{m d}- \\
\Delta \dot{x}_{m}^{T} M_{m} \ddot{x}_{m d}-\dot{x}_{b}^{T} \Gamma_{b m} \dot{x}_{m d}-\Delta \dot{x}_{m}^{T} \Gamma_{m} \dot{x}_{m d}+ \\
\dot{x}_{b}^{T} K_{P b} \Delta x_{b}+\Delta \dot{x}_{m}^{T} K_{P m} \Delta x_{m}
\end{array}
$$

Substituting the control laws (7) and (8) in (14), the timederivative of Lyapunov function results to be:

$$
\dot{V}=-\dot{x}_{b}^{T} K_{D b} \dot{x}_{b}-\Delta \dot{x}_{m}^{T} K_{D m} \Delta \dot{x}_{m} \leq 0 .
$$

As a result of negative semi-definiteness of the time-derivative Lyapunov function in (15), stability is proved. From (15), it is standard to invoke Barbalat's Lemma for non-autonomous systems [22] to conclude that $\dot{\boldsymbol{x}}_{\boldsymbol{b}}, \Delta \dot{\boldsymbol{x}}_{\boldsymbol{m}} \rightarrow 0$ for a trajectory which is bounded in $\ddot{\boldsymbol{x}}_{\boldsymbol{m} \boldsymbol{d}}, \dot{\boldsymbol{x}}_{\boldsymbol{m} \boldsymbol{d}}, \boldsymbol{x}_{\boldsymbol{m} \boldsymbol{d}}$.

For establishing asymptotic stability of such systems, Matrosov theorem has been exploited in literature [9], where an auxiliary function of the states, $W$, is chosen such that $\dot{W} \neq 0$ when $\dot{V}=0$. We choose $W=\Delta \boldsymbol{x}^{T} \boldsymbol{M} \Delta \dot{\boldsymbol{x}}$, which is similar to the one reported in [22] and was used for analysis in joint-space. Computing $\dot{W}$ and substituting the values $M\left[\begin{array}{ll}\ddot{x}_{b}^{T} & \ddot{x}_{m}^{T}\end{array}\right]^{T}$ from (6) with the control laws (7) and (8), we get the following:

$\dot{W}=\Delta \dot{\boldsymbol{x}}^{T} \boldsymbol{M} \Delta \dot{\boldsymbol{x}}+\Delta \boldsymbol{x}^{T}\left(-\boldsymbol{K} \Delta \boldsymbol{x}-\boldsymbol{K}_{\boldsymbol{D}} \Delta \dot{\boldsymbol{x}}+(\dot{\boldsymbol{M}}-\boldsymbol{\Gamma}) \Delta \dot{\boldsymbol{x}}\right)$

where $\boldsymbol{K}_{\boldsymbol{D}}=\left[\left(\boldsymbol{K}_{\boldsymbol{D} \boldsymbol{b}} \mathbf{0}_{6 \times 6}\right)^{T}\left(\mathbf{0}_{6 \times 6} \boldsymbol{K}_{\boldsymbol{D} \boldsymbol{m}}\right)^{T}\right]$. Recalling that the trajectory and the states are bounded, it can be concluded that $\dot{W}$ is bounded. In particular, it can be seen that when $\dot{V} \rightarrow 0, \dot{W}=-\Delta \boldsymbol{x}^{T} \boldsymbol{K} \Delta \boldsymbol{x}$ which is sign-definite (negative) and non-zero for non-zero error $\|\Delta \boldsymbol{x}\|$. Then, with this result, we satisfy the conditions of Matrosov's theorem to establish asymptotic stability of the state in (9).

As validation, we run a simulation with the control laws in (7) and (8). The desired trajectory commanded to the endeffector is shown in Fig. 2 for a position $x$ and orientation $\theta$, where the behaviour of the position trajectory is chosen to prove the efficiency of the controller during tracking.

The tracking error is shown at the top of Fig. 3 and it is expressed in position and orientation as: $\Delta \boldsymbol{x}_{\boldsymbol{m}}=$ $\left(\Delta p_{m}, \Delta \Phi_{m}\right)$. At the bottom, the error $\Delta x_{b}=\left(\Delta p_{b}, \Delta \Phi_{b}\right)$ in position and orientation of the base is shown. As it can be seen, both the errors converge in continuous time. The tracking
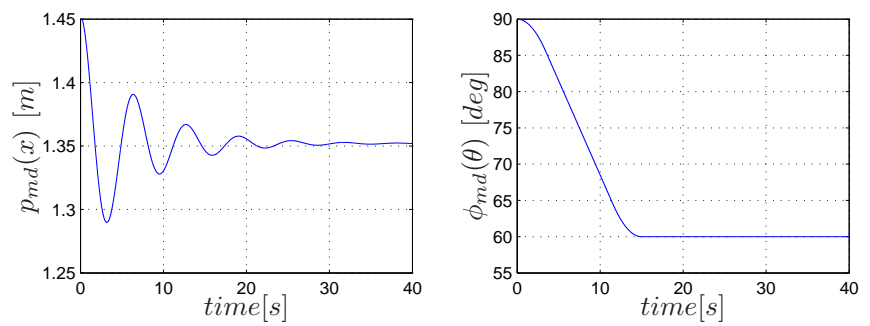

Fig. 2: Desired trajectory for the end-effector, $x_{m d}=\left[\begin{array}{ll}p_{m d}^{T} & \Phi_{m d}^{T}\end{array}\right]^{T}$. 

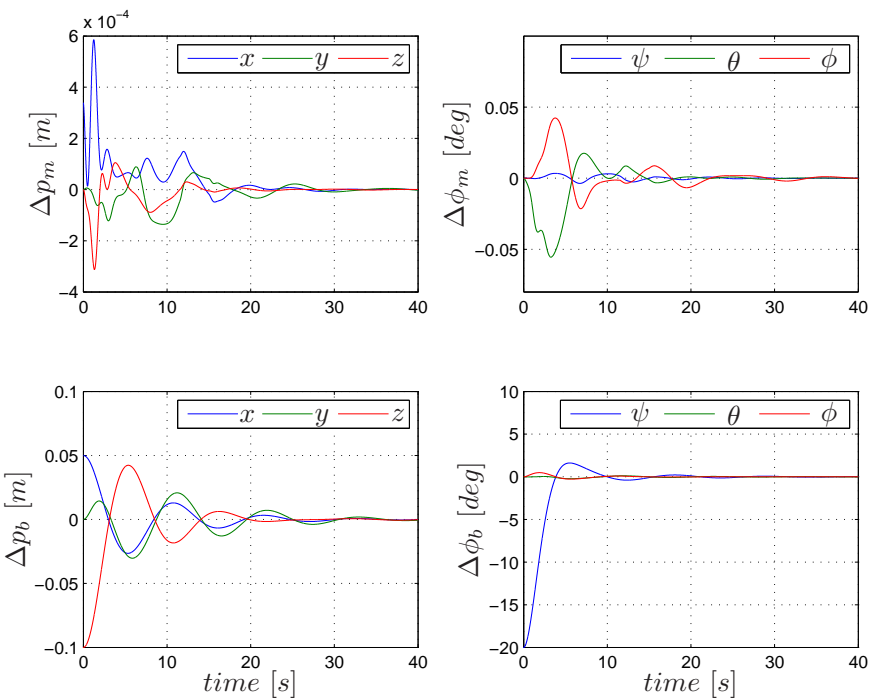

Fig. 3: Stable system with continuous-time controllers. Tracking error of the end-effector (top) and error of the base (bottom).

error is in the order of $10^{-4} \mathrm{~m}$ for the end-effector and the base regulates about the given set-point.

Remark 1: The orientation error is represented using yaw, pitch and roll angles $(\psi, \theta, \phi)$ in the plots of the paper.

\section{B. Coordinated Control in Discrete-time: Problem Statement}

In real space applications, the base is usually actuated with a low rate controller with respect to the manipulator control rate [13]. Let us consider $T_{m}$ to be the sampling time of the controller for the manipulator and $T_{b}$ for the base controller, where $T_{b}=n T_{m}, n \in \mathbb{Z}^{+}$. For a discrete time $k$, it will result that $k=k_{m} T_{m}=k_{b} T_{b}$, where $k_{m}$ and $k_{b}$ are the discrete steps in each controllers. Therefore, (7) and (8) in discretetime can be rewritten as:

$$
\begin{gathered}
F_{m}\left(k_{m}\right)=M_{m}\left(k_{m}\right) \ddot{x}_{m d}\left(k_{m}\right)+\Gamma_{m}\left(k_{m}\right) \dot{x}_{m d}\left(k_{m}\right)- \\
K_{P m} \Delta x_{m}\left(k_{m}\right)-K_{D m} \Delta \dot{x}_{m}\left(k_{m}\right), \quad(16) \\
F_{b}\left(k_{b}\right)=M_{b m}\left(k_{b}\right) \ddot{x}_{m d}\left(k_{b}\right)+\Gamma_{b m}\left(k_{b}\right) \dot{x}_{m d}\left(k_{b}\right)- \\
K_{P b} \Delta x_{b}\left(k_{b}\right)-K_{D b} \dot{x}_{b}\left(k_{b}\right)+J_{b}^{T}\left(k_{b}\right) F_{m}\left(k_{b}\right) . \quad(17)
\end{gathered}
$$

During the inter-sampling period $\left(k_{m}\right.$ and $\left.k_{b}\right)$, the discrete control law might not cancel the respective power terms, as can be seen by substituting $\boldsymbol{F}_{\boldsymbol{m}}\left(\boldsymbol{k}_{\boldsymbol{m}}\right)$ from (16) and $\boldsymbol{F}_{\boldsymbol{b}}\left(\boldsymbol{k}_{\boldsymbol{b}}\right)$ from (17) into (14). Therefore, the discrete nature of the controllers leads to creation of virtual energy which destroys the passivity of the system and it might lead to instability [23].

In the following simulation, the discrete controllers (16) and (17) are applied to the system (6) considering the same initial conditions and trajectory (see Fig. 2) of the continuous case. We assume values of $T_{m}=0.001 \mathrm{~s}$ and $T_{b}=0.3 \mathrm{~s}$, which are typical sampling-times for controllers in space scenarios [13]. The behaviour of the system is shown in Fig. 4. Both the tracking error of the end-effector (top) as well as the error at the base increase (see $\Delta \phi_{b}$ ) and the system results to be unstable. The following section will show how to remove the destabilising effects caused by the discrete controllers.
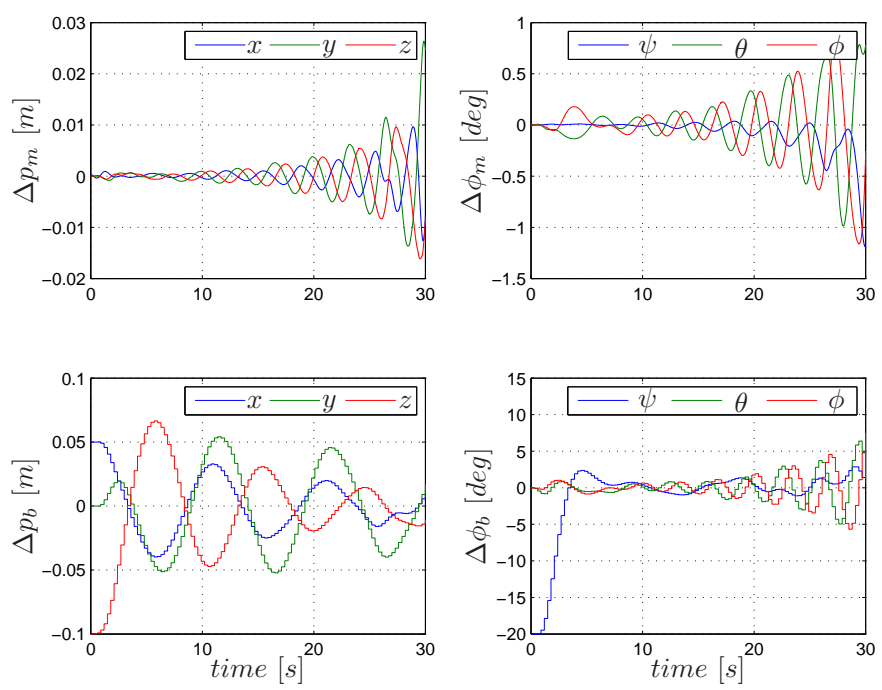

Fig. 4: Unstable system with discrete-time controllers. Tracking error (top) and error of the base (bottom).

\section{Passivity-Based Coordinated Controllers}

This section tackles the strategy adopted for compensating the virtual energy introduced in the system. To this end, Time Domain Passivity Approach (TDPA) is exploited, which will enforce passivity in the discrete controllers by adding a control action based on energy observers [17]. Hence, a background on TDPA is provided in this section and a network representation of the system is proposed in order to design passivity controllers for the base and manipulator.

\section{A. Enforcing Passivity Through TDPA}

Passivity is an input/output characteristic of the system and it is a sufficient condition for achieving stability [24]. For a generic system with power port $(\boldsymbol{F}(\boldsymbol{k}), \boldsymbol{v}(\boldsymbol{k})) \in \mathbb{R}^{n}$, the passivity condition can be expressed as in $[17, \S 2]$ and reported below,

$$
E(m)=E(0)+\sum_{k=0}^{m} \boldsymbol{F}^{\boldsymbol{T}}(\boldsymbol{k}) \boldsymbol{v}(\boldsymbol{k}) T \geq 0,
$$

where $E(0)$ represents the initial energy stored in the system and $T$ is the sampling time. Equation (18) states that the system can not produce energy more than its initial storage and the input energy. Otherwise the system results to be active, i.e. $E(m)<0$, and it will produce energy which can destabilise the system [25]. A common method which exploits the passivity condition in (18) is the TDPA. This approach is based on two elements, a Passivity Observer (PO) which monitors the energy flowing through the power port using (18) and a Passivity Controller (PC), which is activated when the passivity condition is violated (i.e. $E(m)<0$ ). The PC acts with a time-varying damper to dissipate the required amount of energy. Therefore a variable force (or velocity) is commanded to the system to restore the passivity. The benefit of using TDPA is given by the independence on system dynamics knowledge and by the power-port variables available at the network port. 


\section{B. Network Representation for the Manipulator and Base Controllers}

A network representation for the free-flying robot system is shown in Fig. 5 and it will be used for performing energy analysis. Block $S$ represents the dynamics of the free-flying robot, which receives from the left side the forces at the base, i.e. $\boldsymbol{F}_{\boldsymbol{B}}=\boldsymbol{F}_{\boldsymbol{b}}-\boldsymbol{J}_{\boldsymbol{b}}^{\boldsymbol{T}} \boldsymbol{F}_{\boldsymbol{m}}$ and from the right side the controller forces of the manipulator $\boldsymbol{F}_{\boldsymbol{m}}$, as in (16). The two inputs are combined as reported in (6). The network of the base controller is represented with $C_{b}$, which contains the dependent terms $\boldsymbol{F}_{\boldsymbol{b}}^{*}=\boldsymbol{M}_{\boldsymbol{b m}} \ddot{\boldsymbol{x}}_{\boldsymbol{m} \boldsymbol{d}}+\boldsymbol{\Gamma}_{\boldsymbol{b} \boldsymbol{m}} \dot{\boldsymbol{x}}_{\boldsymbol{m} \boldsymbol{d}}$ and the impedance resulting from the stiffness and damping terms, $Z_{c b}$. Similarly, the network for the manipulator controller is represented with $C_{m}$, which contains the dependent term $\boldsymbol{F}_{\boldsymbol{m}}^{*}=\boldsymbol{M}_{\boldsymbol{m}} \ddot{\boldsymbol{x}}_{\boldsymbol{m} \boldsymbol{d}}+\boldsymbol{\Gamma}_{\boldsymbol{m}} \dot{\boldsymbol{x}}_{\boldsymbol{m} \boldsymbol{d}}$ and the impedance of the stiffness and damping terms, $Z_{\mathrm{cm}}$.

Both controllers are represented within dashed boxes in Fig. 5 because they run in discrete-time. In particular, $C_{b}$ runs at a lower rate $T_{b}$ and $C_{m}$ at a higher rate $T_{m}$. This difference in rates while connecting the discrete-controllers to the system dynamics through the ports $B$ and $M$ leads to an unstable system, as discussed in Sec. III-B.

Note that in the design of the controller in continuoustime (Sec. III-A), (10) represents the energy function of the controlled system (interpreting the proportional and the derivative actions of the controllers as springs and dampers). The control actions were able to compensate the coupling between the base and the manipulator, as evident from (15) which prove the stability of the system.

However, since the controllers run at different rates, the compensation of the coupling is not perfect and, therefore it creates virtual energy which destroys the energy balance of the system. Since the system $S$ is passive, if we enforce the passivity of the controllers, the overall controlled system in Fig. 5 given by the interconnection of passive systems is rendered passive. Therefore, the fact that the overall internal energy does not increase, will enforce stability through passivity [24]. By monitoring the energy flows at the ports $B$ and $M$ and, then using passivity controllers, it is possible to ensure the passivity of the overall system. Therefore, we need to define the power correlated variables at the ports $B$ and $M$ of Fig. 5. These can be expressed as (input,output) variables as: $\left(\boldsymbol{F}_{\boldsymbol{B}}, \dot{\boldsymbol{x}}_{\boldsymbol{b}}\right)$ and $\left(\boldsymbol{F}_{\boldsymbol{m}}, \dot{\boldsymbol{x}}_{\boldsymbol{m}}\right)$, respectively. Then, passivity analysis of these ports will be performed in the following subsections by exploiting the TDPA approach.

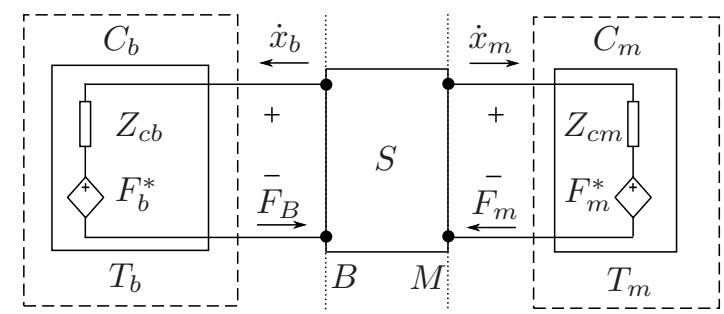

Fig. 5: Network representation of the satellite-manipulator system $(S)$ with tracking controller $C_{m}$ for the manipulator and regulation controller for the base $C_{b}$.

\section{Passivity Observers - Passivity Controllers Architecture}

The passivity observers are designed to monitor the energy of the ports using (18) and will be applied to each degree-offreedom (dof) independently. Enforcing passivity componentwise on the variables composing a port is sufficient for enforcing the passivity of the overall port. In fact, considering (18) and neglecting $E(0)$, the energy can be split into the $n$ components as follows:

$$
\sum_{k=0}^{m}\left(\boldsymbol{F}(k)^{T} \boldsymbol{v}(k) T\right)=\sum_{k=0}^{m} \sum_{i=1}^{n} F_{i}(k) v_{i}(k) T,
$$

where it is evident that if $F_{i}(k) v_{i}(k) T>0$ for each component $i=1, \ldots, n$ then $\sum_{k=0}^{m}\left(\boldsymbol{F}(k)^{T} \boldsymbol{v}(k) T\right)>0$.

1) $\mathrm{PO}-\mathrm{PC}$ for the manipulator controller: A first $\mathrm{PO}-\mathrm{PC}$ is implemented on the port $\left(\boldsymbol{F}_{\boldsymbol{m}}, \dot{\boldsymbol{x}}_{\boldsymbol{m}}\right)$ to monitor the activity of the port $M$. The passivity observer is defined as:

$$
\begin{array}{r}
E_{o b s_{m}}\left(k_{m}\right)=E_{o b s_{m}}\left(k_{m}-1\right)+F_{m}\left(k_{m}\right) \dot{x}_{m}\left(k_{m}\right) T_{m}+ \\
\beta_{m}\left(k_{m}-1\right) \dot{x}_{m}\left(k_{m}-1\right)^{2} T_{m}
\end{array}
$$

where $\beta_{m}$ is the variable damper calculated as:

$$
\beta_{m}\left(k_{m}\right)= \begin{cases}-\frac{E_{o b s_{m}}\left(k_{m}\right)+E_{m}(0)}{\dot{x}_{m}\left(k_{m}\right)^{2} T_{m}} & E_{o b s_{m}}\left(k_{m}\right)<-E_{m}(0) \\ 0 & \text { else. }\end{cases}
$$

with $E_{m}(0)$ being the initial stored energy. The second term on the right side of (20) is the input energy at the port $M$ and the last term is the update of energy which is dissipated by the passivity controller whose force is given by:

$$
F_{p c_{m}}\left(k_{m}\right)=-\beta_{m}\left(k_{m}\right) \dot{x}_{m}\left(k_{m}\right) .
$$

Therefore, if the passivity condition is violated, the correction in force will be provided to the manipulator as follows:

$$
F_{m}^{\prime}\left(k_{m}\right)=F_{m}\left(k_{m}\right)+F_{p c_{m}}\left(k_{m}\right) .
$$

2) $\mathrm{PO}-\mathrm{PC}$ for the base controller: A second PO-PC monitors the port $\left(\boldsymbol{F}_{\boldsymbol{B}}, \dot{\boldsymbol{x}}_{\boldsymbol{b}}\right)$ at the base. In order to have higher accuracy, the passivity observer for this port is implemented in the system running at faster rate $\left(T_{m}\right)$ and it is defined as:

$$
\begin{array}{r}
E_{o b s_{b}}\left(k_{m}\right)=E_{o b s_{b}}\left(k_{m}-1\right)+F_{B}\left(k_{m}\right) \dot{x}_{b}\left(k_{m}\right) T_{m} \\
+\beta_{b}\left(k_{m}-1\right) \dot{x}_{b}\left(k_{m}-1\right)^{2} T_{m},
\end{array}
$$

where the velocity of the base $\dot{x}_{b}$ can be measured at sampling rate $T_{m}$ (eg., using a high-rate position sensor). Notice that the total energy $E_{o b s_{b}}\left(k_{m}\right)$ is updated at each sampling time $T_{m}$. The values of $F_{B}$ changes at each time step $T_{b}$ and between two values, the observer holds the previously received value. Similar to the energy observer for the manipulator, the second term on the right side of (23) is the energy of the port $B$ and the last one is the update of the passivity controller. The variable damper $\beta_{b}$ is derived as:

$$
\beta_{b}\left(k_{m}\right)= \begin{cases}-\frac{E_{o b s_{b}}\left(k_{m}\right)+E_{b}(0)}{\dot{x}_{b}\left(k_{m}\right)^{2} T_{m}} & E_{o b s_{b}}\left(k_{m}\right)<-E_{b}(0) \\ 0 & \text { else, }\end{cases}
$$


where $E_{b}(0)$ is the initial stored energy. Then the force of the passivity controller at the base is calculated as:

$$
F_{p c_{b}}\left(k_{m}\right)=-\beta_{m}\left(k_{m}\right) \dot{x}_{b}\left(k_{m}\right) .
$$

Although the passivity controller is calculated at high rate, its force correction is provided at the sampling step $T_{b}$. Therefore, when the passivity condition is violated, the force provided to the system through the base controller is:

$$
F_{B}^{\prime}\left(k_{b}\right)=F_{b}\left(k_{b}\right)+F_{p c_{b}}\left(k_{b}\right) .
$$

Fig. 6 shows the networks with the PCs placed on the manipulator and base controller. Notice that the force vectors modified by the PO/PC architecture, $F_{B}^{\prime}$ for the base controller and $F_{m}^{\prime}$ for the manipulator controller have been added. Therefore, both the controllers in the dashed boxes endowed with the $P C s$ are rendered passive. Consequently, the overall system is passive since it is an interconnection of passive networks.

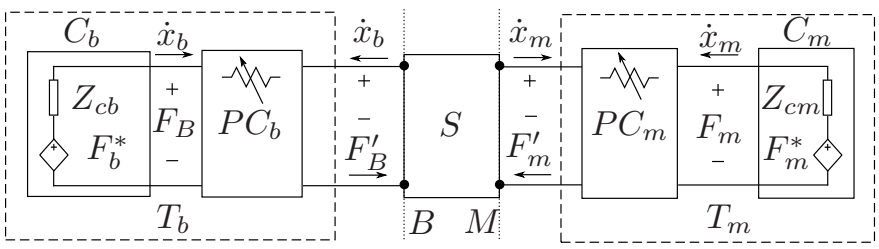

Fig. 6: Network representation scheme with tracking control $C_{m}$ and controller at the base $C_{b}$ endowed with the two PCs.

\section{Simulation Results}

In this section we show the results of the simulation performed with the proposed controllers. The manipulator control runs at $T_{m}=0.001 \mathrm{~s}$ and the base control runs at $T_{b}=0.3 \mathrm{~s}$, which are typical values for a space scenario [13] and we consider the same trajectory as shown in Fig. 2. The base where the manipulator is attached (satellite) has a mass of $M=150 \mathrm{Kg}$ with Inertia $I_{x}=38 \mathrm{kgm}^{2}, I_{y}=20 \mathrm{kgm}^{2}$, $I_{z}=23 \mathrm{kgm}^{2}$ and the mass and inertia parameters of the manipulator are reported in [16].

Fig. 7 shows the energy observed in the system for the manipulator (top) and base (bottom) without the action of the passivity control. Notice that the negative trend indicates activity in the system and it caused the instability showed in Fig. 4. The proposed controller can compensate for this active energy which provides a correction shown in Fig. 8 . The figures at the top show the passivity control action provided to the manipulator and the figures at the bottom, the passivity control of the base. The energy observers with the actions of the PCs are shown in Fig. 9 where the positive semi-definiteness indicates the passivity of the system as per equation (18). Finally the error of the manipulator during the tracking and the error of the base for the regulation are shown in Fig. 10 top and bottom, respectively. It is worth to compare Fig. 4 (before applying the passivity-based method) and Fig. 10 (with the proposed method). It can be seen that all the activity has been removed and the errors converge.
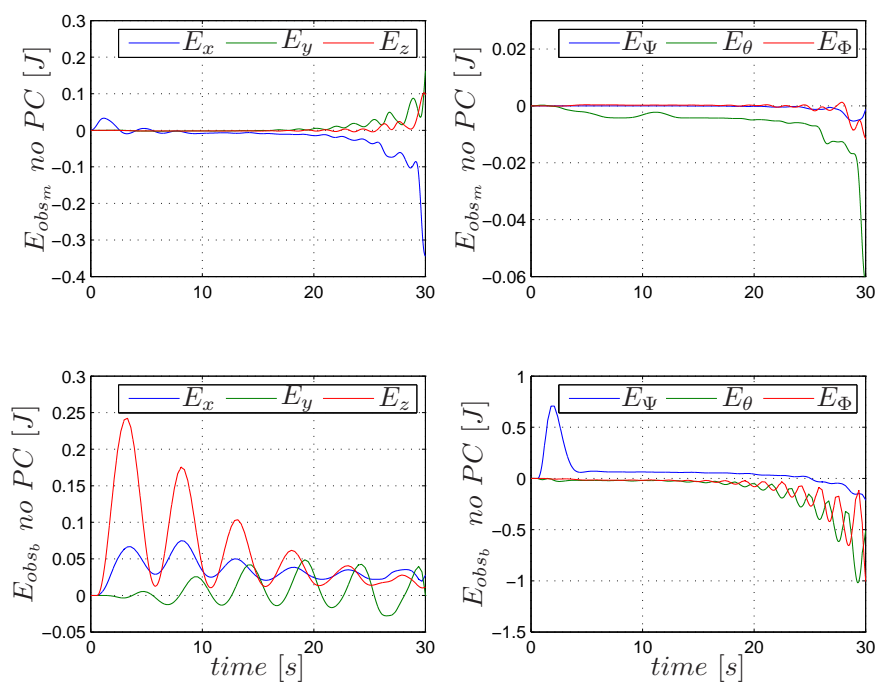

Fig. 7: Energy observers for manipulator (top) and base (bottom) without PC which creates instability shown in Fig. 4.
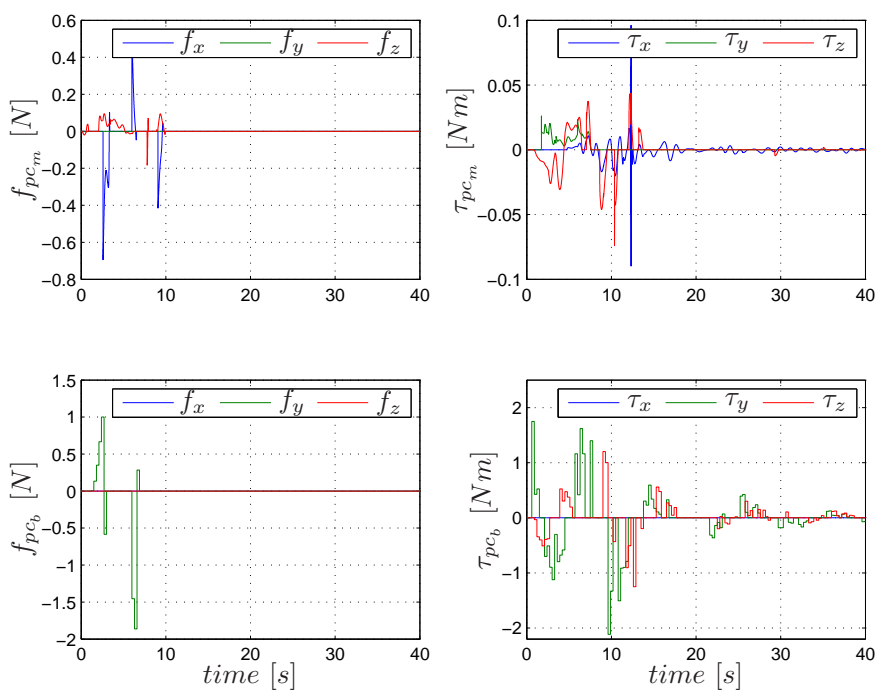

Fig. 8: Passivity controller forces $F_{p c_{m}}=\left(f_{p c_{m}}, \tau_{p c_{m}}\right)$ for the manipulator (top) and passivity controller forces $F_{p c_{b}}=\left(f_{p c_{b}}, \tau_{p c_{b}}\right)$ for the base (bottom).

\section{EXPERIMENT RESULTS}

The coordinated control architecture is validated on a robotic system, the OOS-Sim. The facility exploits industrial robots to simulate satellite dynamics in gravity-free environment. The base motion of the free-flying robot is simulated by the industrial robot shown on the left side of Fig. 1. It moves according to a model-based dynamics as reported in (1). More details about the OOS-Sim facility can be found in [2], [26].

The control of the base runs at $250 \mathrm{~Hz}$ and the manipulator controller at $1000 \mathrm{~Hz}$. The mass and inertial parameters of the space robot are the same as reported in Sec. V. A linear trajectory of $20 \mathrm{~cm}$ is commanded to the Light-Weight-Robot arm along the $-z$ axis in the inertial frame (see Fig. 1) for 10 seconds and the base is regulated about a set-point in a relative position of $[0.04,0,0.08] \mathrm{m}$ and $[10,0,0] \mathrm{deg}$ in orientation with respect to the inertial frame. 

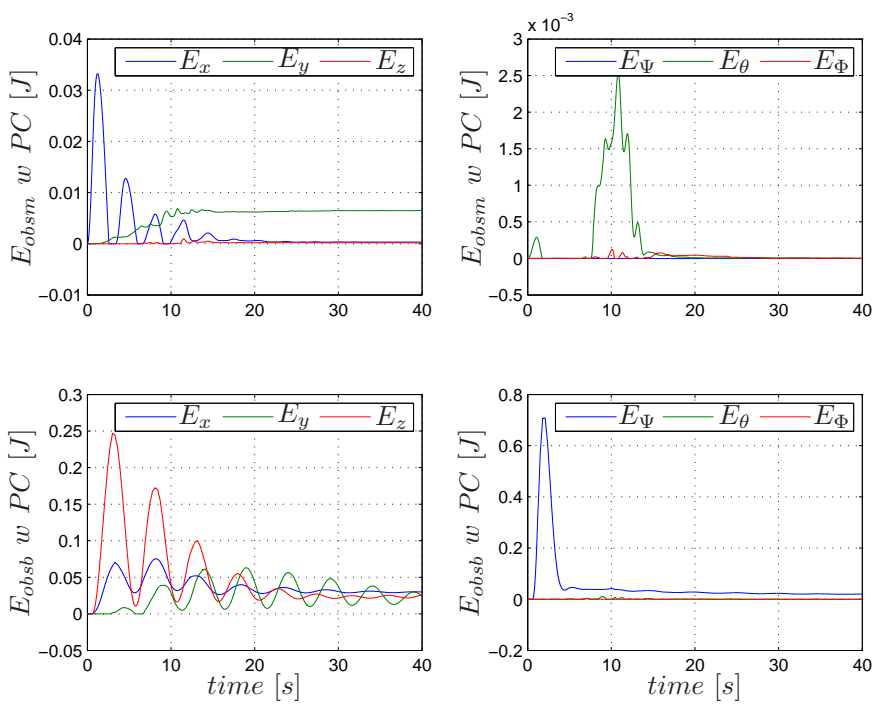

Fig. 9: Energy observers for manipulator (top) and base (bottom) with PC. Positive trends indicate passivity.
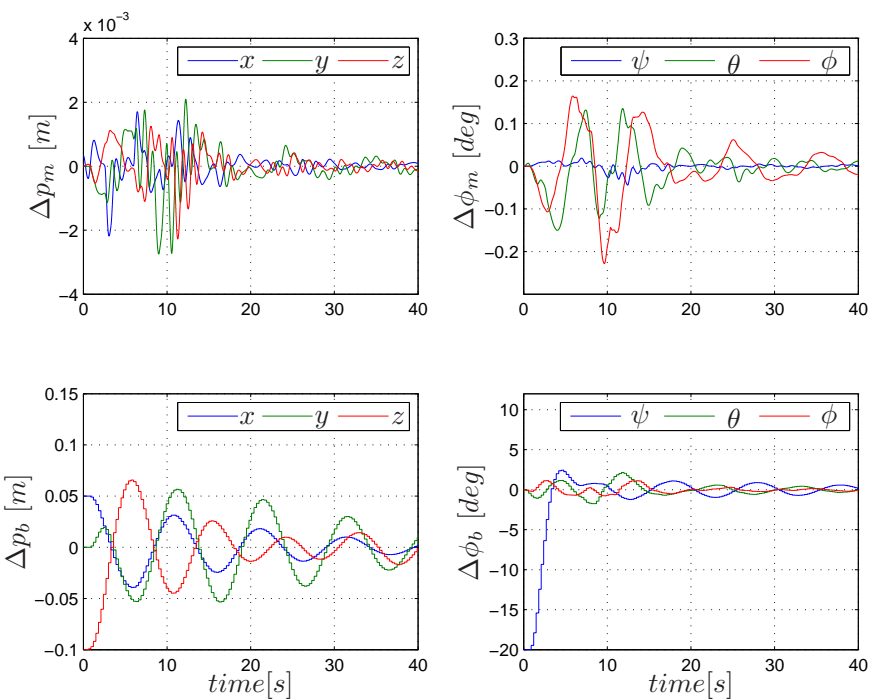

Fig. 10: Tracking error of manipulator (top) and regulation error of the base (bottom) with the proposed controllers.

The tracking error of the manipulator for the position and orientation is shown at the top of Fig. 11 and the force applied are shown at the bottom, respectively. As it can be seen, during the tracking (first $10 \mathrm{~s}$ ) the maximum error is $0.008 \mathrm{~m}$ in position and $1.1 \mathrm{deg}$ in orientation.

The regulation error for the base is shown in Fig. 12 (top) and the respective applied force are shown at the bottom of Fig. 12. The residual error in position and orientation for the base is $0.012 m$ in position and -2 deg in orientation. This is due to the residual forces $\boldsymbol{F}_{\boldsymbol{m}}$ applied by the manipulator and transmitted to the base, as can be seen in (17). During the experiment, the energy observers measured the activity resulting from the multi-rate controllers, as shown in Fig. 13. This extra energy is compensated with the passivity controllers to render the system passive. Fig. 14 shows the energy observers with the action of the passivity controllers. As can be seen, the energy is greater than zero respecting the passivity condition
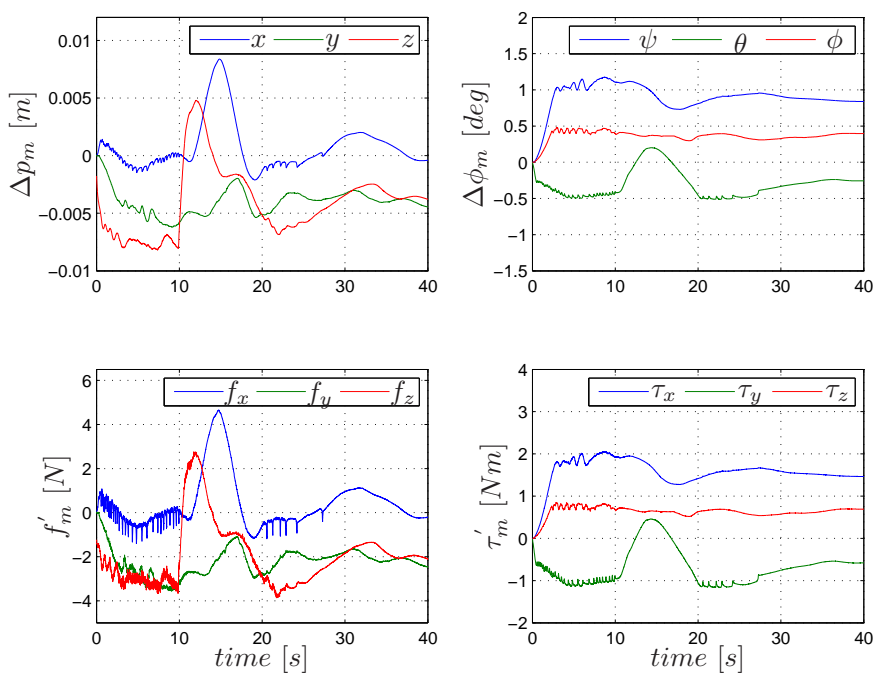

Fig. 11: Experiment results: Tracking error in position and orientation (top) and force $F_{m}^{\prime}=\left(f_{m}^{\prime}, \tau_{m}^{\prime}\right)$ of the manipulator (bottom).
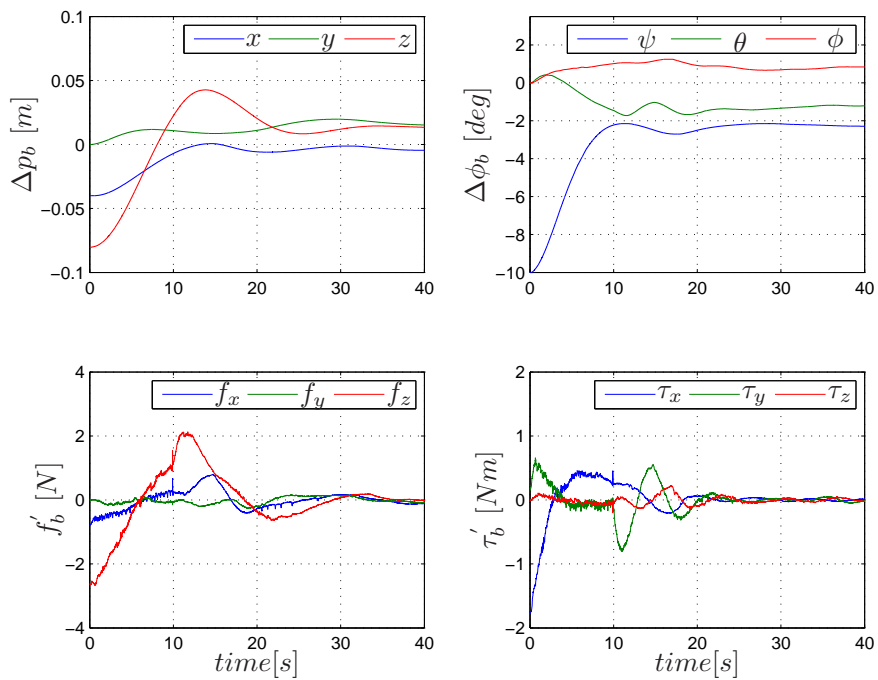

Fig. 12: Experiment results: regulation error of the base (top) and force at the base $F_{B}^{\prime}=\left(f_{b}^{\prime}, \tau_{b}^{\prime}\right)$ (bottom).

in (18). The proposed controllers can run on a real robot in spite of the practical issues like measurement noise, friction and unmodeled dynamics. This and additional experiments can be seen in the video accompanying the paper.

\section{CONCLUSIONS}

Space mission requirements impose hard constraints on the on-board power and relative sampling rates of the controllers. This factor has an effect on the controllers design, which need to fulfill stability characteristics while running at different rates. In this paper we have shown that different sampling rates between two controllers (satellite-base and manipulator) can deteriorate the performances and lead to instability. We proposed a controller architecture endowed with passivity observers and passivity controllers, which can fulfill the task of tracking a desired end-effector trajectory and regulate the base while ensuring stability. The passivity observers have been 

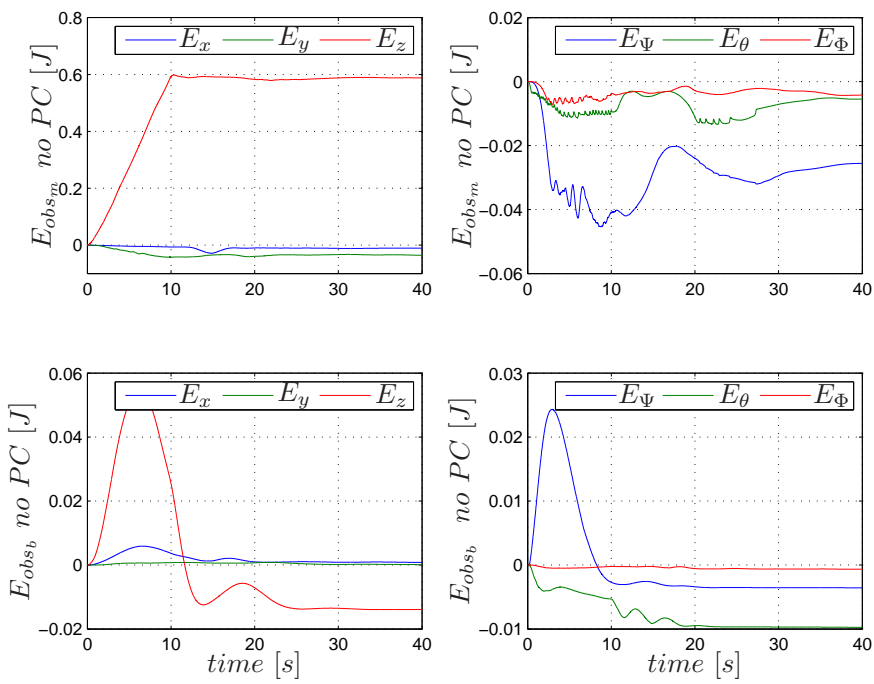

Fig. 13: Experiment results: Energy observers without PC for the manipulator (top) and base (bottom). Negative trend indicates activity.
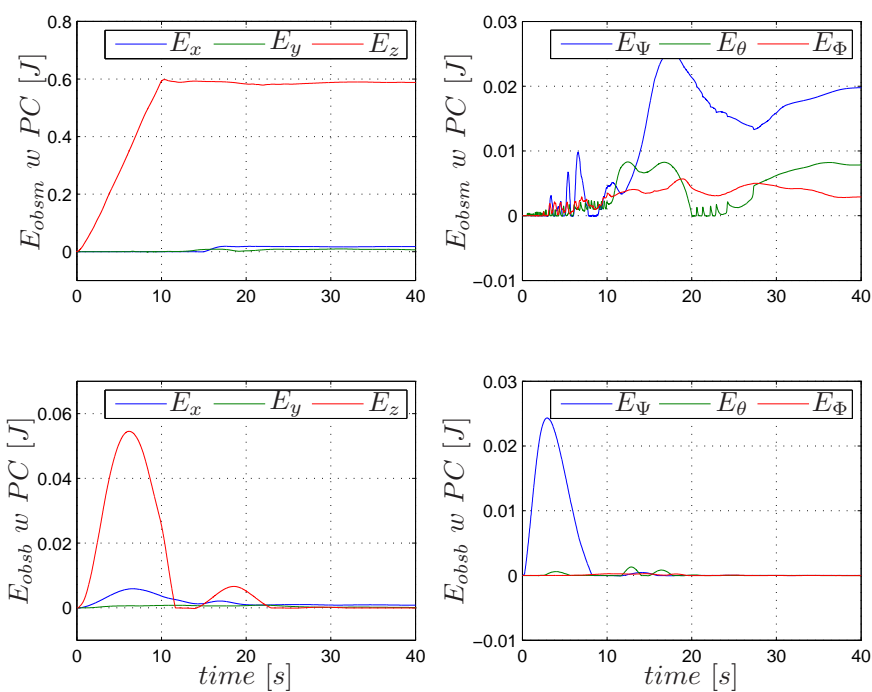

Fig. 14: Experiment results: Energy observers with PC for the manipulator (top) and base (bottom). Positive trend indicates passivity.

designed to monitor the activities of the base and manipulator and two passivity-based controllers have been used for removing the activities. As a result, the overall system is stable. The proposed controllers have been experimentally validated on a robotic facility, the OOS-Sim. The method aims at generality and it can be applied also in other domains.

\section{REFERENCES}

[1] J. Telaar, I. Ahrns, S. Estable, W. Rackl, M. De Stefano, R. Lampariello, N. Santos, P. Serra, C. M., A. F., and J. Gil Fernandez, "Gnc architecture for the e.deorbit mission," 7th Europena Conference for Aeronautics and Space Science (EUCASS), July 2017.

[2] J. Artigas, M. De Stefano, W. Rackl, R. Lampariello, B. Brunner, W. Bertleff, R. Burger, O. Porges, A. Giordano, C. Borst, and A. AlbuSchaeffer, "The OOS-SIM: An on-ground simulation facility for on-orbit servicing robotic operations," in Robotics and Automation (ICRA), IEEE International Conference on, May 2015, pp. 2854-2860.

[3] H. Nakanishi and K. Yoshida, "Impedance control for free-flying space robots -basic equations and applications-," in 2006 IEEE/RSJ Interna- tional Conference on Intelligent Robots and Systems, Oct 2006, pp. $3137-3142$.

[4] K. Nanos and E. G. Papadopoulos, "On the dynamics and control of free-floating space manipulator systems in the presence of angular momentum," Frontiers in Robotics and AI, vol. 4, p. 26, 2017.

[5] Y. Umetani and K. Yoshida, "Resolved motion rate control of space manipulators with generalized jacobian matrix," Robotics and Automation, IEEE Transactions on, vol. 5, no. 3, pp. 303-314, Jun 1989.

[6] Y. Masutani, F. Miyazaki, and S. Arimoto, "Sensory feedback control for space manipulators," in Proceedings, 1989 International Conference on Robotics and Automation, May 1989, pp. 1346-1351 vol.3.

[7] K. Yoshida, "Practical coordination control between satellite attitude and manipulator reaction dynamics based on computed momentum concept," in IEEE/RSJ/GI International Conference on Intelligent Robots and Systems '94., vol. 3, sep 1994, pp. $1578-1585$.

[8] E. Papadopoulos and S. Dubowsky, "Coordinated manipulator/spacecraft motion control for space robotic systems," in Robotics and Automation, IEEE International Conference on, Apr 1991, pp. 1696-1701 vol.2.

[9] B. Paden and R. Panja, "Globally asymptotically stable pd+ controller for robot manipulators," International Journal of Control, vol. 47, no. 6, pp. 1697-1712, 1988

[10] R. Lampariello, H. Mishra, N. Oumer, P. Schmidt, M. De Stefano, and A. Albu-Schffer, "Tracking control for the grasping of a tumbling satellite with a free-floating robot," IEEE Robotics and Automation Letters, vol. 3, no. 4, pp. 3638-3645, Oct 2018.

[11] J. R. Spofford and D. L. Akin, "Redundancy control of a free-flying telerobot," Journal of Guidance, Control, and Dynamics, vol. 13, no. 3, pp. $515-523,1990$.

[12] "e.deorbit implementation plan," Clean Space, European Space Research and Technology Centre, ESA Report ESA-TEC-SC-TN-2015-007, Dec. 2015.

[13] J. Telaar, S. Estable, M. De Stefano, W. Rackl, R. Lampariello, F. Ankersen, and J. Gil Fernandez, "Coupled control of chaser platform and robot arm for the e.deorbit mission," 10th Int. ESA conference on Guidance Navigation and Control Systems (GNC), May 2017.

[14] V. Tanasa, S. Monaco, and D. Normand-Cyrot, "Backstepping control under multi-rate sampling," IEEE Transactions on Automatic Control, vol. 61, no. 5, pp. 1208-1222, May 2016.

[15] N. Diolaiti, G. Niemeyer, F. Barbagli, and J. Salisbury, "Stability of haptic rendering: Discretization, quantization, time delay, and coulomb effects," Robotics, IEEE Transactions on, vol. 22, no. 2, pp. 256-268, April 2006.

[16] M. De Stefano, R. Balachandran, A. Giordano, C. Ott, and C. Secchi, "An energy-based approach for the multi-rate control of a manipulator on an actuated base," in Robotics and Automation (ICRA), 2018 IEEE International Conference on, May 2018, pp. 1072-1077.

[17] J.-H. Ryu, D.-S. Kwon, and B. Hannaford, "Stability guaranteed control: Time domain passivity approach," IEEE Transactions on Control Systems Technology, vol. 12, no. 6, pp. 860-868, 2004.

[18] M. De Stefano, J. Artigas, and C. Secchi, "An optimized passivity-based method for simulating satellite dynamics on a position controlled robot in presence of latencies," in 2016 IEEE/RSJ International Conference on Intelligent Robots and Systems (IROS), Oct 2016, pp. 5419-5426.

[19] M. De Stefano, R. Balachandran, J. Artigas, and C. Secchi, "Reproducing physical dynamics with hardware-in-the-loop simulators: A passive and explicit discrete integrator," in Robotics and Automation (ICRA), 2017 IEEE International Conference on, May 2017, pp. 5899-5906.

[20] R. Featherstone, Rigid Body Dynamics Algorithms. Secaucus, NJ, USA: Springer-Verlag New York, Inc., 2007.

[21] J. E. Slotine and W. Li, Eds., Applied nonlinear control. Englewood Cliffs (N.J.) Prentice Hall, 1991.

[22] A. Loria, E. Panteley, D. Popovic, and A. R. Teel, "A nested matrosoy theorem and persistency of excitation for uniform convergence in stable nonautonomous systems," IEEE Transactions on Automatic Control, vol. 50, no. 2, pp. 183-198, Feb 2005.

[23] S. Stramigioli, C. Secchi, A. J. van der Schaft, and C. Fantuzzi, "Sampled data systems passivity and discrete port-hamiltonian systems," IEEE Transactions on Robotics, vol. 21, no. 4, pp. 574-587, Aug. 2005.

[24] A. van der Schaft, $L_{2}$-Gain and Passivity Techniques in Nonlinear Control, ser. Communication and Control Engineering. Springer, 2000.

[25] C. Secchi, S. Stramigioli, and C. Fantuzzi, Control of Interactive Robotic Interfaces: a port-Hamiltonian Approach, ser. Springer Tracts in Advanced Robotics. Springer, 2007.

[26] M. De Stefano, J. Artigas, A. Giordano, R. Lampariello, and A. AlbuSchaeffer, "On- ground experimental verification of a torque controlled free-floating robot," in 13th Symposium on Advanced Space Technologies in Robotics and Automation 2015 (ASTRA), May 2015. 